\title{
Magneto-optical Behaviors at a 2-D Ferromagnetic/Organic Semi- conductor Interface for Singlet Fission
}

\author{
Jeremy T. Tisdale, Mingxing Li, Yu-Che Hsiao, Ting Wu, and Bin $\mathrm{Hu}^{*}$
}

\begin{abstract}
This article reports the magneto-optical effects on the singlet fission of the p-type organic semiconductor, tetracene, from a ferromagnetic/semiconductor interface between thin films of cobalt and tetracene. We experimentally show that this interface has two effects on the thin films of tetracene: spin interactions and electrical polarization. The experimental tools used to study the interface include magnetic field effect photoluminescence $\left(\mathrm{MFE}_{\mathrm{PL}}\right)$, photoluminescence and absorption. Spin interaction effects are shown by $\mathrm{MFE}_{\mathrm{PL}}$ data, where we observe a large increase in the maximum MFE $\mathrm{PL}_{\mathrm{PL}}$ when cobalt is introduced, as well as changes in the hyperfine interactions at low magnetic fields. Electrical polarization is analyzed with photoluminescence and absorption measurements, showing small changes in the energy difference between the HOMO and LUMO levels of tetracene, as well as an increase in the electron-phonon coupling in tetracene. Also, electrical polarization is shown to increase electrical interactions between tetracene molecules. Therefore, we conclude that using spin interactions and electrical polarization from the ferromagnetic/organic semiconductor interface can tune the properties of tetracene, ultimately enhancing singlet fission. This work gives new insight to understand the singlet fission process using a ferromagnetic interface. These changes can be further utilized in photovoltaic applications based on this singlet fission material and be applied to other similar types of singlet fission organic semiconductors.
\end{abstract}




\section{GRAPHICAL ABSTRACT}

Electrical polarization and spin interactions due to the interface between cobalt and tetracene cause a change in the conversion rate between triplet pairs. This leads to an increase in triplet state densities, which causes an increase in singlet fission of tetracene.

(a)

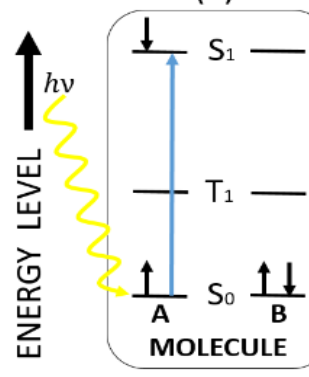

(b)

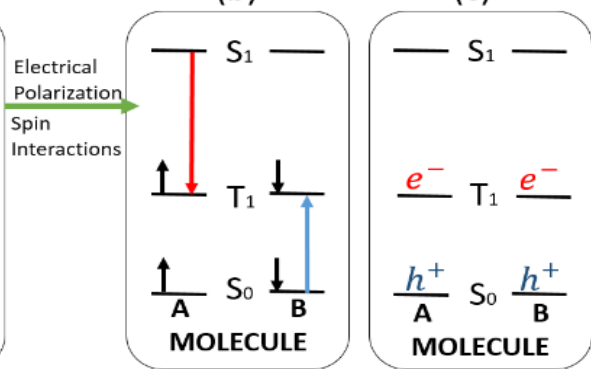

\section{INTRODUCTION}

Organic systems have gained high attention for photovoltaics as the efficiency of such systems have rapidly grown from $4.4 \%$ in 2004 up to $11 \%$ in 2014 (an increase of $10 \%$ over a span of only 10 years)..$^{1,2}$ Organic photovoltaics (OPVs) have many promising properties for use in solar cells including, but not limited to: flexibility, transparency, thin active layers, low cost and easy fabrication. On the other hand, the theoretical power conversion efficiency (PCE) of bulk heterojunction solar cells has been determined to be about $23 \% .{ }^{3}$ In order to further improve the efficiencies and abilities for commercial application of OPVs, it is required to study and understand the basic fundamentals behind the mechanisms in organic semiconducting materials that allows for photon conversion to electricity. Singlet fission is a process in certain organic semiconductors that has been a breakthrough towards higher PCEs of organic solar cells. Singlet exciton fission, or singlet fission, is a spin-dependent process in which one singlet excited state converts to two triplet excited states. By this process, it has been shown that organic semiconductors are able to absorb one photon, consequently generating two excited triplet states..$^{4-10}$ During singlet fission, a photon is absorbed from the UV-VIS light spectrum. This photon excites one molecule of the organic semiconductor from the ground singlet state $\left(\mathrm{S}_{0}\right)$ to an excited singlet state $\left(\mathrm{S}_{1}\right)$. The excited singlet state then relaxes to a preferred excited triplet state $\left(\mathrm{T}_{1}\right)$ via intersystem crossing. Electronic coupling between neighboring molecules then allows for the energy from the relaxation of the first molecule to be shared by resonance energy transfer. The energy transfer excites the neighboring molecule from the $S_{0}$ state to the $T_{1}$ state. Each excited triplet state results in a free electron-hole pair. Tetracene has been heavily studied as a prospect material for OPVs because of its singlet fission process. When singlet fission is paired with a solar cell with suitable low energy gap materials, it has been shown to increase power conversion efficiency by enhancing photocurrent in the visible spectrum. $\stackrel{11}{ }$ Tetracene has been shown to be one of the best candidates for singlet fission due to strong triplet-triplet interactions, which is why it has been chosen as the studied system for this work. The triplet yield of tetracene has been shown to reach $153 \% \pm 5 \%$, generating internal quantum efficiencies (IQE) of $127 \% \pm 18 \%$. Here, IQE is defined as how many excited triplet states are generated per one photon absorbed. Interfacial interactions are heavily studied for device processing for many different applications including photovoltaic devices. A p-n heterojunction is one of the well-known interfaces used in organic photovoltaic devices. For example, a tetracene(p-type)/ $\mathrm{C}_{60}$ (n-type), donor/acceptor interface is widely used in tetracenebased organic photovoltaics. $\frac{8-11}{}$ Many other types of interfaces have been used in photovoltaic applications to improve desired properties of devices. The main interface this work focuses on is the interface between a ferromagnetic layer and an organic semiconducting layer. Many studies have been done on the beneficial effects from a ferromagnetic/organic semiconducting interface. $\frac{12-18}{\text { It }}$ is apparent from the 
process that singlet fission is a spin-dependent process. Therefore, the most important properties that need to be studied to understand singlet fission in tetracene and at the interface are spin interactions, which directly affect conversion rates between triplet states, and electrical polarization at the interface. These properties are studied to control singlet fission using magnetic field effects.

\section{EXPERIMENTAL SECTION}

Tetracene and cobalt wire were purchased from Sigma Aldrich and used as received for sample preparation. The pre-cleaned glass substrates were treated with UV-zone before sample preparation. For thin films without cobalt, tetracene films were deposited on the glass substrates by thermal evaporation with thicknesses of 10, 15, 50,100, and $150 \mathrm{~nm}$. For thin films with cobalt, cobalt was first deposited on the glass substrates by thermal evaporation with a thickness of $10 \mathrm{~nm}$, then the same tetracene thicknesses from above were deposited onto the cobalt. All thermal evaporation was done in a high-vacuum chamber at a pressure of $2 \times 10^{-6}$ torr. All measurements in these experiments were done at room temperature. Absorption data was collected after sample preparation before any other measurements to avoid interference from photoexcitation. Photoluminescence (PL) and magneto-photoluminescence (MFE $\mathrm{PL}_{\text {) were }}$ measured using a Fluorolog spectrofluorometer from Horiba and a $405 \mathrm{~nm} \mathrm{CW}$ laser for the photoexcitation source. During $\mathrm{MFE}_{\mathrm{PL}}$ measurements, samples were placed between poles of a large electromagnet parallel to the applied magnetic field of 0-900 $\mathrm{mT}$, while the photoexcitation source was aligned at around a $60^{\circ}$ angle to the sample. $\mathrm{MFE}_{\mathrm{PL}}$ is defined in Equation 1 as

$$
M F E_{P L}=\frac{P L_{B}-P L}{P L}
$$

where $\mathrm{PL}_{\mathrm{B}}$ is the photoluminescence under the applied magnetic field and PL is the base photoluminescence with no applied magnetic field. During $\mathrm{MFE}_{\mathrm{PL}}$, results were reproducible for multiple samples and reported from the first time the external magnetic field was applied. This was done to ensure that the magnetization memory of the cobalt thin film did not influence the results. Measurements were also repeated on same samples to ensure magnetization memory had no effects on the $\mathrm{MFE}_{\mathrm{PL}}$ results.

\section{RESULTS \& DISCUSSION}

$\mathrm{MFE}_{\mathrm{PL}}$ has been shown to be an important experimental tool to elucidate information regarding the singlet fission rate of a material by studying magnetic field effects on the inter-conversion rate between ${ }^{5}(\mathrm{TT})$ and ${ }^{1}(\mathrm{TT})$, triplets with quintet characteristics and singlet characteristics, respectively. It is well known that $\mathrm{MFE}_{\mathrm{PL}}$ can be used to elucidate information about singlet fission. ${ }^{7}$ In this work, we have demonstrated the use of $\mathrm{MFE}_{\mathrm{PL}}$ to characterize singlet fission in thin films of tetracene and investigate how spin and electrical interactions affect singlet fission. First, we show that electrical interactions between triplet states is thickness dependent in thin films (between 10 and $150 \mathrm{~nm}$ ), whereby increasing the thickness of the film increases the amount of molecules in the film. Because of this, the probability that electrical interactions occur between excited molecules and unexcited molecules increases, increasing the amount of possible triplet states and enhancing singlet fission. We then show that singlet fission can be enhanced further using a thin semi-transparent layer of cobalt as a ferromagnetic substrate. We demonstrate this by showing a large $\mathrm{MFE}_{\mathrm{PL}}$ increase when the cobalt/tetracene interface is introduced to the system. Because, the large increases are only shown by thin tetracene films, $(10 \mathrm{~nm})$ we verify that the spin-interactions between cobalt and tetracene occur at the interface. Singlet fission rate is determined by the density of ${ }^{1}$ (TT) states. At low fields, hyperfine interactions can enhance inter-conversion between triplet pair states. Therefore, we see a decrease in $\mathrm{MFE}_{\mathrm{PL}}$, which means there is an increase in ${ }^{1}$ (TT) states and an increase in singlet fission. At high fields, spin-exchange interactions are high, disallowing inter-conversion between triplet states. Therefore, we see an increase in the positive MFE $\mathrm{PL}_{\mathrm{am}}$ 
plitude, which shows that the density of ${ }^{5}$ (TT) is high meaning that singlet fission rate is low. However, this decrease in singlet fission rate is caused by disallowing conversion from the external magnetic field. When the high external magnetic field is not applied, the inter-conversion will return to normal allowing for singlet fission. Adding the cobalt layer is shown to increase the amplitude of both the hyperfine and spin-exchange regime. These phenomena are caused by spin interactions at the interface, which cause an increase in the conversion of triplet states, resulting in an increase in singlet fission. Figure 1(a) shows the percent change of magneto-photoluminescence $\left(\mathrm{MFE}_{\mathrm{PL}}\right)$ for tetracene thin films ranging from $10 \mathrm{~nm}$ to $150 \mathrm{~nm}$ with and without cobalt. The line shape of each sample shows Lorentzian characteristics in the negative components, agreeing with previous studies. ${ }^{7}$
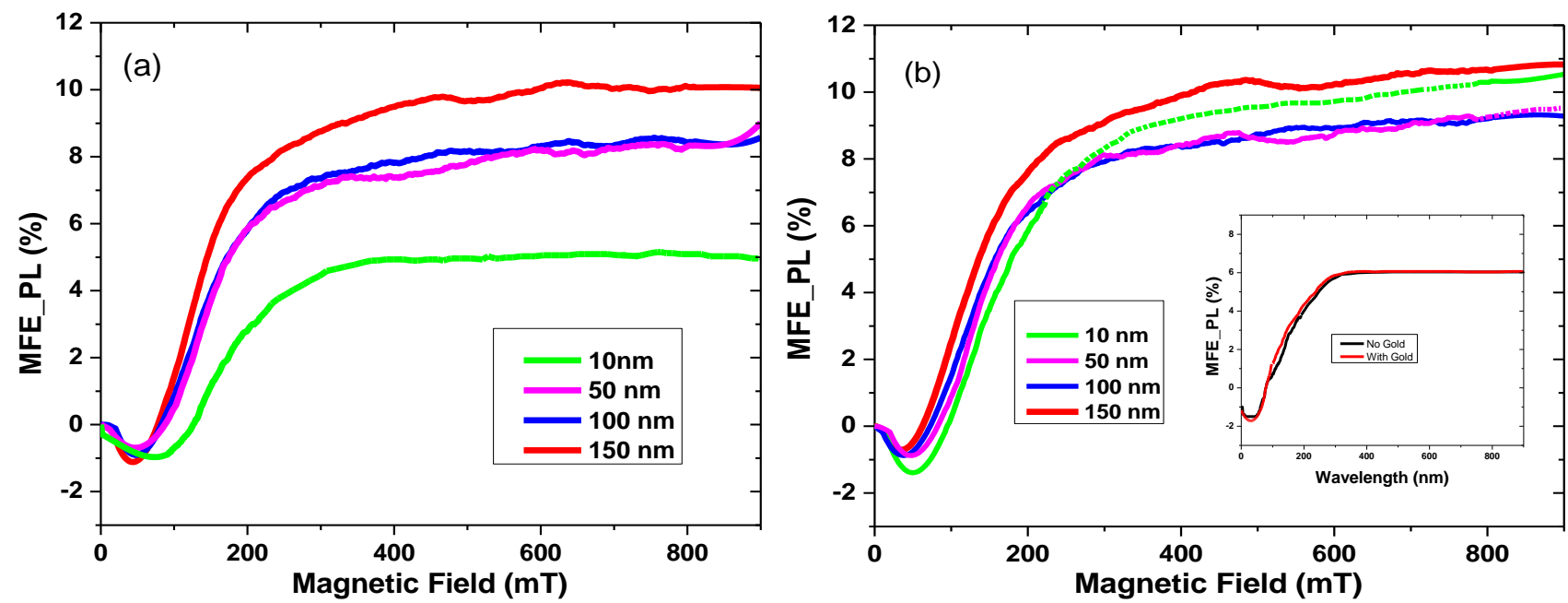

Figure 1: Magneto-photoluminescence for Tetracene Sample with Thicknesses of 10, 50, 100 and 150 $\mathrm{nm}$ (a) without a Cobalt Layer and (b) with a $10 \mathrm{~nm}$ Layer of Cobalt between the Tetracene and Glass. (Inlet of Figure 1(b) shows same measurement for $10 \mathrm{~nm}$ film of tetracene with and without a $10 \mathrm{~nm}$ gold film in place of the cobalt layer.)

All of the films from $50 \mathrm{~nm}$ to $150 \mathrm{~nm}$ film thickness in Figure 1 have an initial negative MFE $\mathrm{PL}_{\mathrm{c}}$ component at a low field $(<50 \mathrm{mT})$ and then a positive $\mathrm{MFE}_{\mathrm{PL}}$ signal at a higher field $(>50 \mathrm{mT})$. The $10 \mathrm{~nm}$ film shows higher zero-crossing point critical field values as compared to the thicker films. In Figure 1(a), there is a clear trend in thickness dependence for $\mathrm{MFE}_{\mathrm{PL}}$, where increasing the thickness of the tetracene thin film increases the maximum percent change in photoluminescence. At an applied magnetic field of $900 \mathrm{mT}$, the $150 \mathrm{~nm}$ film of tetracene reaches a maximum change of $10.0 \%$, the $100 \mathrm{~nm}$ and $50 \mathrm{~nm}$ films reach a maximum change of $8.6 \%$ and $9.0 \%$, respectively, and the $10 \mathrm{~nm}$ film reaches a maximum change of $5.0 \%$. These maximum values of $\mathrm{MFE}_{\mathrm{PL}}$ can be analyzed to determine the relative density of triplet pair states formed in the films of tetracene (higher $\mathrm{MFE}_{\mathrm{PL}}$ maximum means increased density in triplet states). Therefore, it is shown that when the thickness of tetracene is increased, the density of triplet states increases. Once the magnetic field is no longer applied and inter-conversion between triplet states can occur at a normal rate. Therefore, it is shown that the thicker films will have more triplet states available for inter-conversion resulting in increased singlet fission rate. Figure 1(b) shows the same measurements as Figure 1(a) with the addition of a $10 \mathrm{~nm}$ cobalt layer. It is shown at an applied magnetic field of $900 \mathrm{mT}$, the $150 \mathrm{~nm}$ film of tetracene reaches a maximum change of $10.8 \%$, and the $100 \mathrm{~nm}$ and $50 \mathrm{~nm}$ films reach a maximum change of 9.3\% and 9.5\%, respectively. Comparing Figure 1(b) to Figure 1(a), there are only small changes in $\mathrm{MFE}_{\mathrm{PL}}$ maximums $(0.5-0.8 \%)$ for thicker tetracene films of tetracene $(50 \mathrm{~nm}$ to $150 \mathrm{~nm})$ when cobalt is added. Also, the same thickness dependence from the $50 \mathrm{~nm}$ to $150 \mathrm{~nm}$ tetracene films is shown with and without cobalt. This shows that inter- 
facial interactions between tetracene and cobalt do not have much of an impact on thicker films of the organic semiconductor. This shows that interactions between molecules inside thicker films of tetracene are dominant over interfacial interactions, forbidding the interfacial interactions to have large impacts of the material properties. However, for the $10 \mathrm{~nm}$ tetracene film, there are major changes in the MFE $\mathrm{PL}_{\mathrm{L}}$ in the large-field spin exchange regime and low-field hyperfine when the $10 \mathrm{~nm}$ cobalt layer is added. Under a high magnetic field, the maximum $\mathrm{MFE}_{\mathrm{PL}}$ change increases by $5.5 \%$ to a maximum value of $10.5 \% \mathrm{MFE}_{\mathrm{PL}}$ change. This phenomenon shows that adding the $10 \mathrm{~nm}$ cobalt layer to the system is able to greatly increase the density of triplet pair states. Here, the data shows direct evidence that the inherent magnetic field of cobalt causes spin interactions at the interface, which increases the conversion between triplet pair states. There is also a line shape change in the low-field range from 0 to $200 \mathrm{mT}$ for the $10 \mathrm{~nm}$ tetracene film. Here the negative component and the zero-crossing point change in $\mathrm{MFE} \mathrm{PL}_{\mathrm{PL}}$ can be used to gain understanding of the relative strength of the hyperfine interactions in tetracene compared to the strength of interfacial interactions. At a low field, each electron experiences a slightly different field strength because of hyperfine interactions. These interactions are seen by the negative component of the $\mathrm{MFE}_{\mathrm{PL}}$, whereby spin mixing generated by hyperfine interactions causes an increase in the inter-conversion of triplet pair states. From the negative $\mathrm{MFE}_{\mathrm{PL}}$ component at low field, we can see that the critical magnetic field strength required to disturb the hyperfine interactions-based spin mixing becomes lower. This means that the spin interactions at the interface between cobalt and tetracene facilitate aligning the spins of electrons with the applied magnetic field. Therefore, a smaller external magnetic field is required to eliminate spin mixing due to hyperfine interactions. When the cobalt layer is added to the system the critical applied magnetic field for the zero crossing point of the MFE $\mathrm{PL}_{\text {decreas- }}$ es from $126 \mathrm{mT}$ to $95 \mathrm{mT}$. This data confirms that adding a thin ferromagnetic layer between the glass and tetracene reduces the effects of hyperfine interactions at low external magnetic fields. Also, at low fields, it is shown that adding a cobalt film increases the negative amplitude of $\mathrm{MFE}_{\mathrm{PL}}$ from $1 \%$ to $1.5 \%$ showing an increase in singlet fission. The inlet of Figure 1(b) shows the same photoluminescence measurements with and without a $10 \mathrm{~nm}$ film of gold for a $10 \mathrm{~nm}$ film of tetracene. This shows that there are no significant changes in the low field or high field regime when the $10 \mathrm{~nm}$ gold layer is added. This data shows that the changes caused by cobalt are true phenomena of the cobalt/tetracene interface as explained above.
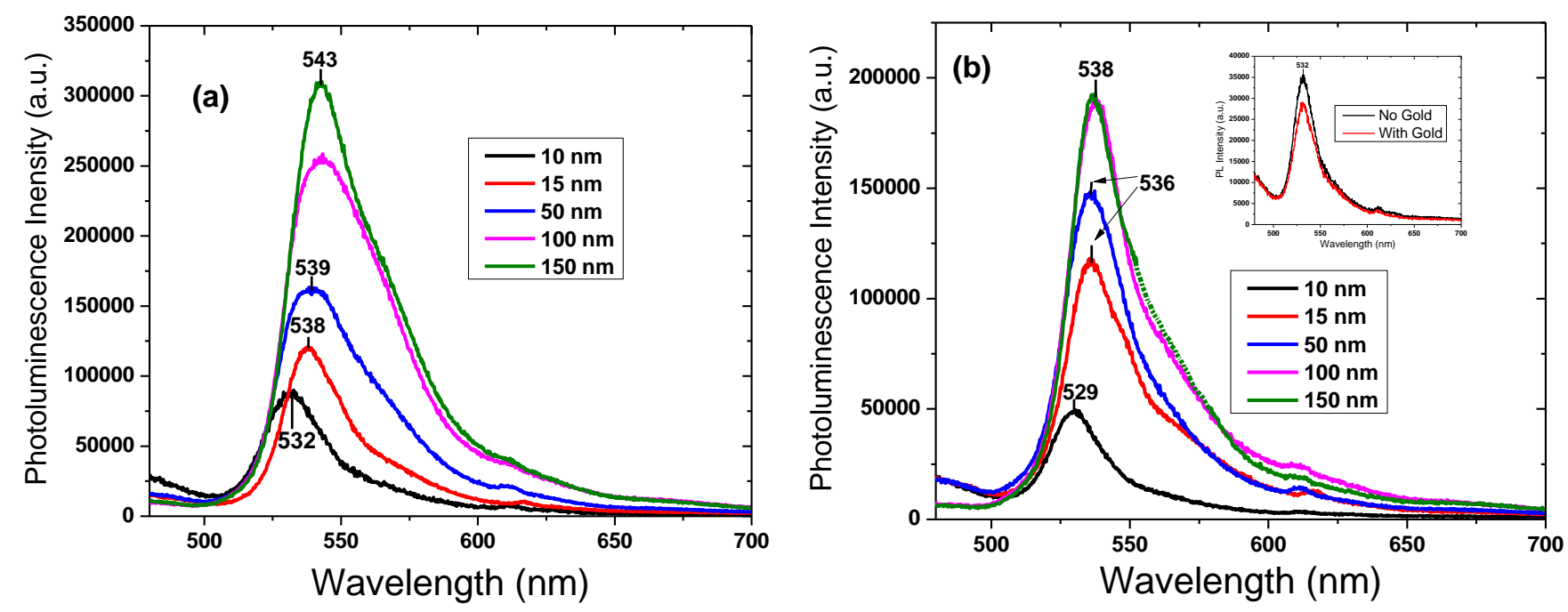

Figure 2: Photoluminescence Spectra for Tetracene Samples with Thicknesses of 10, 15, 50, 100 and 150 nm (a) without a Cobalt Layer and (b) with a $10 \mathrm{~nm}$ Cobalt Layer Between the Tetracene and Glass. (Inlet of Figure 2(b) shows same measurement for 10 $\mathrm{nm}$ film of tetracene with and without a $10 \mathrm{~nm}$ gold film in place of the cobalt layer.) 
Photoluminescence and absorption measurements were also used to study the interactions related to electrical polarization at the ferromagnetic/organic semiconductor interface. From photoluminescence measurements it was determined that the energy difference between the HOMO and LUMO levels in the tetracene thin films increased as the thickness of the thin films decreased. It is shown that the cobalt/tetracene substrate caused a blue shift in the photoluminescence spectra. From this phenomenon, it is clear to see that the blue shifts are caused by electrical polarization at the interface. From the evidence that electrical polarization occurs at the interface, we can conclude that the electrical polarization enhances electrical interactions between molecules in tetracene further enhancing singlet fission. Figure 2 shows the photoluminescence spectra of tetracene thin films with varying thicknesses ranging from 10 $\mathrm{nm}$ to $150 \mathrm{~nm}$. Figure 2(a) shows data for samples without a cobalt layer, while Figure 2(b) shows data for samples with a $10 \mathrm{~nm}$ layer of cobalt between the glass substrate and tetracene thin film. In both cases, it is shown that photoluminescence intensity decreases with decreasing thickness of the tetracene layer. This is because the thinner films of tetracene are not able to absorb as much photoexcitation and have less molecules to be excited than thicker films. It can also be seen in both cases that decreasing the thickness of the tetracene layer causes a blue shift in the photoluminescence peak. This is shown by the blue shifts in PL with decreasing film thicknesses. It is known that decreasing the thickness of a thin film semiconductor will increase the energy difference between the HOMO and LUMO levels in organic semiconductors. $\stackrel{19,20}{ }$ Comparing Figure 2(a) to 2(b), it can be seen that adding the thin layer of cobalt between the glass and tetracene also causes a blue shift $(\sim 3-5 \mathrm{~nm}$ depending on thickness of tetracene layer) in the photoluminescence peak. The effect of electrical polarization when semiconducting materials are in contact with metallic materials has been greatly studied. $\underline{21} \underline{22}$ From this data, it is evident that the ferromagnetic/semiconducting interface causes electrical polarization on the thin tetracene thin film. We also note that adding a cobalt layer shows quenching in the PL. This further confirms that electrical polarization occurs at the interface between cobalt and tetracene, as quenching of PL is caused by the following two effects from the interface. One effect leading to quenching of PL is energy transfer, whereby excitons in tetracene recombine and the released energy from recombination is absorbed by cobalt instead of being released as fluorescence. The other effect leading to quenching is charge transfer, whereby cobalt is able to donate electrons to the tetracene layer. In order to understand the charge transfer direction, the energy levels of tetracene and cobalt are important. The HOMO and LUMO levels of tetracene are $5.4 \mathrm{eV}$ and $2.7 \mathrm{eV}$, respectively. The work function of cobalt in $5.0 \mathrm{eV}$. From this information, it can be noted that charge transfer occurs from tetracene to cobalt at the interface. The donated electrons are able to pair with the holes of excitons leading to dissociation of excitons. The dissociation of excitons results in quenching of PL as the dissociated excitons will no longer recombine to give off fluorescence. The charge transfer from cobalt to tetracene will then cause a dipole to be formed at the interface, where cobalt becomes positively charged after donating electrons. The inlet of Figure 2(b) shows the same photoluminescence measurements with and without a $10 \mathrm{~nm}$ film of gold for a $10 \mathrm{~nm}$ film of tetracene. The difference in PL intensity for when gold substrate is added, is explained by photoluminescence quenching due to the organic/metallic interface charge transfer. However, this data shows that there is no blue shift in photoluminescence when the $10 \mathrm{~nm}$ gold layer is added, shows that the changes caused by cobalt are true phenomena as explained above, rather than simple causes such as morphological changes due to changing the substrate.

By analyzing the absorption data, it is clear that the electrical polarization at the interface causes an increase in electron-phonon coupling in thin tetracene thin films. Figure 3 shows the absorption spectra of tetracene thin films with and without a $10 \mathrm{~nm}$ layer of cobalt. Figure 3(a) shows the data for a $10 \mathrm{~nm}$ thin film of tetracene, while Figure 3(b) shows the data for a $100 \mathrm{~nm}$ thin film of tetracene. The absorption data for the tetracene thin films without cobalt are in agreement with previous results. $\underline{5}$ 

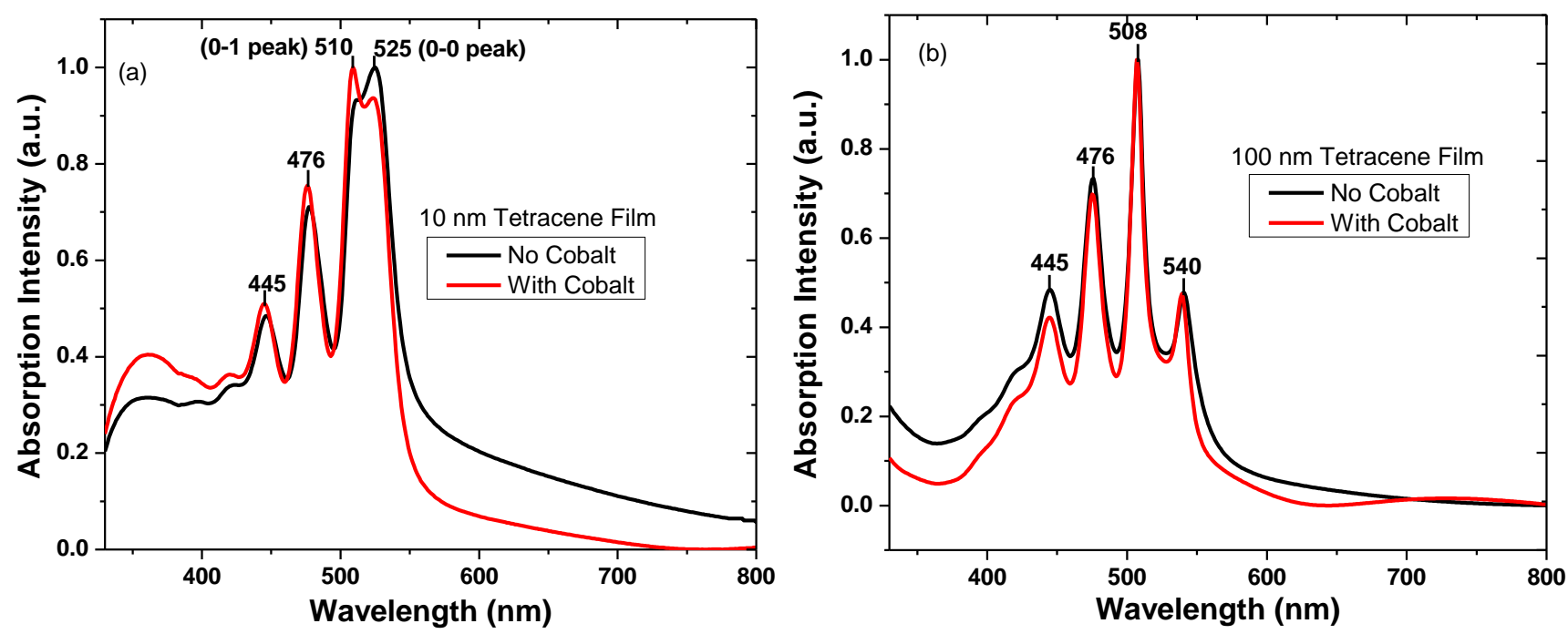

Figure 3: Absorption Spectra for Tetracene Samples with and without a $10 \mathrm{~nm}$ Cobalt Layer between the Glass and Tetracene for (a) a $10 \mathrm{~nm}$ Layer of Tetracene and (b) a $100 \mathrm{~nm}$ Layer of Tetracene.

By comparing Figures 3(a) and 3(b), it is shown that the $525 \mathrm{~nm}$ peak from the $10 \mathrm{~nm}$ tetracene sample shifts from the $540 \mathrm{~nm}$ peak from the $100 \mathrm{~nm}$ tetracene sample. This shift in absorption data is caused by a change in band gap due to thin film thickness, as previously discussed. This blue shift in absorption also agrees with the blue shift in photoluminescence. In Figure 3(b), there are small, negligible changes in the absorption spectrum when cobalt is added. This is due to the fact that interfacial interactions do not have a large impact on thicker films of tetracene. However, in Figure 3(a) there is a clear change in relative peak intensity in the 510 and $525 \mathrm{~nm}$ peaks. The relative peak intensity change is due to electrical polarization at the interface. The relative peak intensity change can be studied to understand changes in electron-phonon coupling inside the tetracene thin film. Because the electron-phonon coupling trend is shown in the absorption data in Figure 3(a), the $510 \mathrm{~nm}$ peak is labeled as the $0-1$ peak and the 525 $\mathrm{nm}$ peak is labeled as the $0-0$ peak. It is shown that the $0-1$ peak is weaker than the $0-0$ peak in tetracene without cobalt. When cobalt is added to the system, the $0-1$ peak becomes much stronger than the $0-0$ absorption peak in tetracene. This analysis shows that the electrical polarization in tetracene caused by the electrical interfacial interactions with cobalt increases the electron-phonon coupling in the sample. Increases in the electronic-phonon coupling is bad for photovoltaic applications, as it makes charge transfer more difficult. Therefore, for photovoltaic application it is best to find a way to limit energy loss by making exciton dissociation a quicker process than energy loss to electron phonon coupling.

\section{CONCLUSIONS}

In summary, this work explored the magneto-optical effects on the organic semiconductor tetracene from the interfacial interactions between itself and the ferromagnetic layer, cobalt. We used magnetophotoluminescence, photoluminescence and absorption to study the interfacial interactions and found that the interface can act upon the system in two ways: spin interactions and electrical polarization. Spin interactions at the interface caused an increase in the conversion rate of triplet pairs, enhancing the singlet fission of tetracene. Electrical polarization at the interface is able to increase the electrical interactions between molecules, also enhancing singlet fission. Electrical polarization also caused an increase in the HOMO/LUMO energy difference in tetracene. Also, due to electrical polarization at the interface, we observed quenching of photoluminescence and an increase in electron-phonon coupling. Therefore, we see that using the ferromagnetic layer can cause interfacial interactions between cobalt and tetracene enhancing singlet fission towards enhancing its photovoltaic capabilities. Further studies can be done to 
ensure that the cobalt layer inside a solar cell device will enhance power conversion efficiencies due to the enhanced singlet fission of tetracene.

\section{AUTHOR INFORMATION}

\section{Corresponding Author}

Dr. Bin $\mathrm{Hu}$

(865) $974-3946$

bhu@utk.edu

\section{Notes}

The authors declare no competing financial interest.

\section{ACKNOWLEDGMENT}

We thank the Center for Materials Processing (J.T.), a Center of Excellence at the University of Tennessee, Knoxville funded by the Tennessee Higher Education Commission (THEC), for financial support. This research was supported by the financial supports from Air Force Office of Scientific Research (AFOSR) (FA 9550-15-1-0o64) and National Science Foundation (CBET1438181).

\section{ABBREVIATIONS}

$\mathrm{MFE}_{\mathrm{PL}}$, Magnetic field effect photoluminescence; OPV, Organic photovoltaic; PCE, Power conversion energy; IQE, Internal quantum efficiency

\section{REFERENCES}

(1) Li, G.; Shrotriya, V.; Huang, J.; Yao, Y.; Moriarty, T.; Emery, K.; Yang, Y., High-Efficiency Solution Processable Polymer Photovoltaic Cells Be Self-Organization of Polymer Blends. Nature Materials 2005, 4, 864-868.

(2) Green, M. A.; Emery, K.; Hishikawa, Y.; Warta, W.; Dunlop, E. D., Solar Cell Efficiency Tables (Version 45). Progress in Photovoltaics: Research and Applications 2015, 23, 1-9.

(3) Kirchartz, T.; Taretto, K.; Rau, U., Efficiency Liits of Organic Bulk Heterojunction Solar Cells. Journal of Physical Chemistry C 20o9, $113,17958-17966$.

(4) Burdett, J. J.; Bardeen, C. J., The Dynamics of Singlet Fission in Crystalline Tetracene and Covalent Analogs. Accounts of Chemical Research 2013, 46, 1312-1320.

(5) Burdett, J. J.; Muller, A. M.; Gosztola, D.; Bardeen, C. J., Excited State Dynamics in Solid and Monomeric Tetracene: The Roles of Superradiance and Exciton Fission. The Journal of Chemical Physics 2010, 133, 1-12.

(6) Tayebjee, M.; Clady, R.; Schmidt, T., The Exciton Dynamics in Tetracene Thin Films. Physical Chemistry Chemical Physics 2013, 15, 14797-14805.

(7) Qiao, X.; Luan, L.; Liu, Y.; Yu, Z.; Hu, B., Inter-Triplet Spin-Spin Interaction Effects on Inter-Conversion between Different Spin States in Intermediate Triplet-Triplet Pairs Towards Singlet Fission. Organic Electronics 2014, 15, 2168-72.

(8) Wu, T. C.; Thompson, N. J.; Congreve, D. N.; Hontz, E.; Yost, S. R.; Voorhis, T. V.; Baldo, M. A., Singlet Fission Efficiency in Tetracene-Based Organic Solar Cells. Applied Physics Letters 2014, 104, 1-4.

(9) Priya, J. J.; Mohanty, A.; Sussman, J.; Lee, J.; Baldo, M. A., Singlet Exciton Fission in Nanostructured Organic Solar Cells. Nano Letters 2011, 11, 1495-1498.

(10) Lee, J.; Jadhav, P.; Reusswig, P. D.; Yost, S. R.; Thompson, N. J.; Congreve, D. N.; Hontz, E.; Voorhis, T. V.; Baldo, M. A., Singlet Exciton Fission Photovoltaics. Account of chemical research 2012, 46, 1300-1311.

(11) Chu, C.; Shao, Y.; Shrotriya, V.; Yang, Y., Efficient Photovoltaic Energy Conversion in Tetracene-C6o Based Heterojunctions. Applied Physics Letters 2005, 86, 1-3.

(12) Annese, E.; Casolari, F.; Fujii, J.; Rossi, G., Interface Magnetic Coupling of Fe-Phthalocyanine Layers on a Ferromagnetic Surface. Physical Review $B$ 2013, 87, 1-6.

(13) Hsu, Y.; Lai, Y. L.; Chen, C.; Lin, Y.; Chien, H.; Wang, J.; Lam, T.; Chan, Y.; Wei, D. H.; Lin, H., et. al., Enhanced Magnetic Anisotropy Via Quasi-Molecular Magnet at Organic-Ferromagnetic Contact. The Journal of Physical Chemistry Letters 2013, 4, 310-316.

(14) Li, M.; He, L.; Xu, H.; Shao, M.; Tisdale, J.; Hu, B., Interaction between Optically-Generated Charge-Transfer States and Magnetized Charge-Transfer States toward Magneto-Electric Coupling. The Journal of Physical Chemistry Letters 2015, 6, 4319-4325.

(15) Moorsom, T.; Wheeler, M.; Khan, T. M.; Ma'Mari, F. A.; Kinane, C.; Langridge, S.; Ciudad, D.; Bedoya-Pinto, A.; Hueso, L.; Teobaldi, G., et. al., Spin-Polarized Electron Transfer in Ferromagnet/C6o Interfaces. Physical Review B 2014, 90, 1-6.

(16) Raman, K. V.; Kamerbeek, A. M.; Mukherjee, A.; Atodiresei, N.; Sen, T. M.; Lazic, P.; Caciuc, V.; Michel, R.; Stalke, D.; Mandal, S. K., et. al., Interface-Engineered Templates for Molecular Spin Memory Devices. Nature 2013, 493, 509-513.

(17) Steil, S.; Grossmann, N.; Laux, M.; Ruffing, A.; Steil, D.; Wiesenmayer, M.; Mathis, S.; Monti, O. L. A.; Cinchetti, M.; Aeschlimann, M., Spin-Dependent Trapping of Electrons at Spinterfaces. Nature Physics 2013, 9, 1-6.

(18) Vita, H.; Bottcher, S.; Leicht, P.; Horn, K.; Shick, A. B.; Maca, F., Electronic Structure and Magnetic Properties of Cobalt Intercalated in Graphene on $\operatorname{Ir}(111)$. Physical Review B 2014, 90, 1-10. 
(19) Das, N. S.; Ghosh, P. K.; Mitra, M. K.; Chattopadhyay, K. K., Effect of Film Thickness on the Energy Band Gap of Nanocrystalline Cds Thin Films Analyzed by Spectroscopic Ellipsometry. Physica E 2010, 42, 2097-2102.

(20) Rabeh, M. B.; HKhedmi, N.; Fodha, M. A.; Kanzari, M., The Effect of Thickness on Optical Band Gap and N-Type Conductivity of Cuins2 Thin Films Annealed in Air Atmosphere. Energy Procedia 2014, 44, 52-60.

(21) Pluchery, O.; Zhang, Y.; Benbalagh, R.; Caillard, L.; Gallet, J. J.; Bournel, F.; Lamic-Humbolt, A. F.; Salmeron, M.; Chabal, Y. J.; Rochet, F., Static and Dynamic Electron Characterization of Organic Monlayers Rafted on a Silicon Surface. Royal Society of Chemistry 2016, 18, 3675-3684.

(22) Wang, Z.; Cao, D.; Wen, L.; Xu, R.; Obergfell, M.; Mi, Y.; Zhan, Z.; Nasori, N.; Demsar, J.; Lei, Y., Manipulation of Charge Transfer and Transport in Plasmonic-Ferroelectric Hybrids for Photoelectrochemical Applications. Nature Communications $2015,7,1-8$. 
Electrical polarization and spin interactions due to the interface between cobalt and tetracene cause a change in the conversion rate between triplet pairs. This leads to an increase in triplet state densities, which causes an increase in singlet fission of tetracene.

(a)

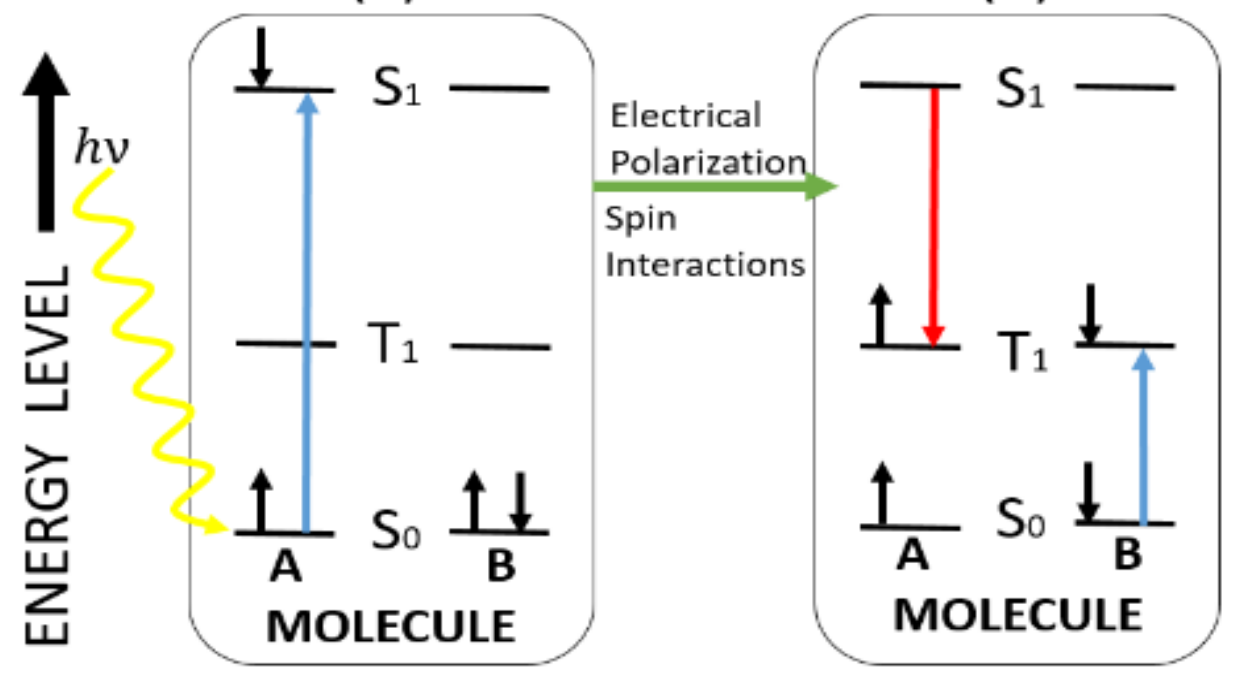

(c)

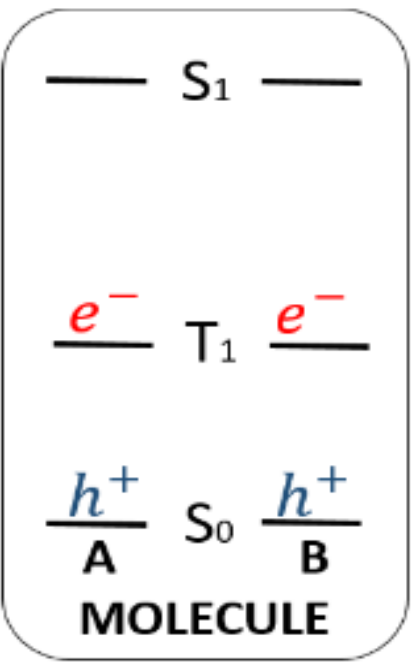

